FIT-901

hep-th/99xxxxx

\title{
Born-Infeld strings between D-branes
}

\author{
K. Ghoroku ${ }^{a}$ 円 and K. Kaneko ${ }^{b}$ ? \\ ${ }^{a}$ Department of Physics, Fukuoka Institute of Technology, \\ Wajiro Higashi-ku 811-02, Fukuoka, Japan \\ ${ }^{b}$ Department of Physics, Kyushu Sangyo University \\ Matsukadai, Higashi-ku, 813-8503, Fukuoka, Japan
}

\begin{abstract}
We examine the solutions of world-volume action for a D3-brane being put near other D3-brane which is replaced by the background configuration of bulk space. It is shown that the BPS solutions are not affected by the D3brane background, and they are interpreted as dyonic strings connecting two branes. On the contrary, the non-BPS configurations are largely influenced by the background D-brane, and we find that the solutions with pure electric charge cannot connect two branes. These solutions are corresponding to the bound state of brane and anti-brane which has been found by Callan and Maldacena.
\end{abstract}

\footnotetext{
${ }^{1}$ gouroku@fit.ac.jp

${ }^{2}$ kaneko@phys.kyusan-u.ac.jp
} 


\section{Introduction}

Recently, classical solutions of the Born-Infeld (BI) action, which is considered as the world-volume action of the D-brane, have been studied [1, 2, 3, 田 in the flat background-space. They are classified into the BPS and non-BPS solutions. The stable BPS solutions are interpreted as the strings which connect two D-branes separated by the infinite distance. While the non-BPS solutions are not protected from the dynamical fluctuations since they are not supersymmetric one. Among them, non-BPS solutions with pure electric charge have attracted an attention [1]. It can be regarded as the half-part of the string which connects brane and anti-brane with a finite distance to form a bound state of them. The stability of such a non-BPS configuration has been studied from a viewpoint of the quantum mechanics [1, 5].

It will be interesting to examine these solutions from a viewpoint of the $S U(N)$ Yang-Mills theory which can be constructed by the stack of D3-branes. In this direction, some progress has been given by studying the world-volume action of test brane embedded in the background of D-branes [6, 7, 8, 9, 10]. The situation of the symmetry breaking $S U(N) \rightarrow S U(N-1) \times U(1)$ is realized by setting one of the branes far away from the others. Then we can say that the classical solutions of the world-volume action represent the soliton solutions such as monopoles or dyons appearing in the non-Abelian Yang-Mills theory. It would be meaningful to study the non-BPS solutions which are non-supersymmetric solution obtained in the background of the type-IIB superstring theory, because they are expected to play some role in the non-supersymmetric Yang-Mills theory.

The purpose of this paper is to study the classical solutions of the world-volume action in a situation that the test D-brane is set parallel to a background D-brane(s). In the next section, the model is given. And in section 3.1, we show the BPS and non-BPS dyonic solutions in the flat background. In sections 3.2 and 4, we solve the case of the D-brane background. We find that the BPS solutions are not affected by the background, but the configuration of non-BPS solutions are influenced by the background. Especially, the pure electric solutions cannot reach at the position of the background brane(s). Conclusions are given in the final section.

\section{D-brane action to be solved}

Even if we consider the supersymmetric case, the fermionic coordinates are not necessary to obtain a classical solution of the D-brane action. So we neglect them, and the bosonic part of the effective action of a D-p-brane being coupled to the background can be written as follows,

$$
S_{p+1}=-T_{p} \int d^{p+1} \xi\left(\mathrm{e}^{-\phi} \sqrt{-\operatorname{det}\left(G_{\mu \nu}+F_{\mu \nu}\right)}+\frac{1}{(p+1) !} \epsilon^{i_{1} \cdots i_{p+1}} A_{i_{1} \cdots \cdots i_{p+1}}\right)
$$

where $T_{p}$ denotes the tension of the D-p-brane and

$$
F_{\mu \nu}=\partial_{\mu} A_{\nu}-\partial_{\nu} A_{\nu}
$$

is the field strength of $U(1)$ gauge field living in the world volume. We also neglect

here the antisymmetric tensor $B_{M N}$, which should be added to $F_{\mu \nu}$, since the brane 
considering here has no NS-NS charge. The metric $G_{\mu \nu}$ and the $(p+1)$-form are the pullback of the space-time metric $G_{M N}$ and the R-R $(p+1)$-form respectively,

$$
\begin{gathered}
G_{\mu \nu}=G_{M N} \partial_{\mu} X^{M} \partial_{\nu} X^{N} \\
A_{i_{1} \cdots \cdots i_{p+1}}=\partial_{i_{1}} X^{M_{1}} \cdots \partial_{i_{p+1}} X^{M_{p+1}} A_{M_{1} \cdots M_{p+1}} .
\end{gathered}
$$

The embedding of the world volume into the target space is described by $X^{M}\left(\xi^{\mu}\right)$ as a function of the $p+1$ dimensional world-volume coordinates $\xi^{\mu}$.

Our purpose is to solve the equations of motion for the above action $S_{p+1}$ under the target space configuration for $\Phi, G_{M N}$ and $A_{M_{1} \cdots M_{p+1}}$, which are obtained as the D-p-brane configuration by solving the ten-dimensional supergravitational theory. Before solving the equations of D-brane action in this way, we briefly review the D-brane configurations.

They are obtained by solving the supergravity effective action corresponding to the superstring theory [11, 12]. The bosonic part of the low energy effective action in $d$-dimensional pl target space can be written as

$$
S_{\mathrm{d}}=-\int d^{d} x \sqrt{-g}\left\{\mathrm{e}^{2 \Phi}\left(R+4(\partial \Phi)^{2}-\frac{1}{12} H_{3}^{2}\right)-\frac{1}{2(p+2) !} F_{p+2}^{2}\right\}
$$

where $H_{3}(=d B)$ is the three form field strength of the antisymmetric tensor $B_{M N}$ and $F_{p+2}\left(=d A_{p+1}\right)$ denotes the $(p+2)$-form field-strength of the $(p+1)$-form $\mathrm{R}-\mathrm{R}$ potential $A_{p+1}$.

In solving the classical equations of $S_{d}$ with respect to $g_{M N}, \Phi$ and $A_{p+1}$, several ansatzs are adopted. The $d$-dimensional coordinates $x^{M}$ are denoted by separating them into the tangential $\left(x^{\mu}\right)$ and the transverse part $\left(y^{m}\right)$ to the D-p-brane as $x^{M}=\left(x^{\mu}, y^{m}\right)$, where $\mu=0 \sim p$ and $m=p+1 \sim d-1$. Then take the following ansatzs

$$
\begin{gathered}
d s^{2}=\mathrm{e}^{2 A} \eta_{\mu \nu} d x^{\mu} d x^{\nu}+\mathrm{e}^{2 B} \delta_{m n} d y^{m} d y^{n}, \\
A_{p+1}=-\mathrm{e}^{C} d x^{0} \wedge d x^{1} \wedge \cdots \wedge d x^{p},
\end{gathered}
$$

where $A, B, C$ and $\Phi$ are the functions of $y$ only, here $y=\sqrt{\delta_{m n} y^{m} y^{n}}$. The last statement means that the solutions are assumed to be invariant under $P_{p+1} \otimes S O(d-$ $p-1)$ transformation. Then $A, B, C$ and $\Phi$ are all expressed by a common function, $H(y)$, which is determined by the field equations derived by $S_{d}$. The final result is obtained as follows:

$$
\begin{gathered}
\mathrm{e}^{2 A(y)}=H(y)^{-1 / 2}, \quad \mathrm{e}^{2 B(y)}=H(y)^{1 / 2}, \\
\mathrm{e}^{C(y)}=H(y)^{-1}-1, \quad \mathrm{e}^{2 \Phi}=H(y)^{(3-p) / 2},
\end{gathered}
$$

where

$$
\begin{gathered}
H(y)=1+2 Q \kappa T_{p} G(y), \\
G(y)=\left\{\begin{array}{cc}
{\left[\tilde{d}|y| \tilde{d} \Omega_{\tilde{d}+1}\right]^{-1}} & \tilde{d}>0 \\
-\frac{1}{2 \pi} \log |y| & \tilde{d}=0 .
\end{array}\right\},
\end{gathered}
$$

${ }^{3}$ Here $d$ is used to denote the target space dimension, but we consider the case of $d=10$ throughout this paper 
and $\tilde{d}=d-p-3$. Here $\Omega_{q}=2 \pi^{(q+1) / 2} / \Gamma((q+1) / 2)$ denotes the area of a unit $q$-dimensional sphere $S^{q}$.

Here $B_{M N}=0$ since the D-brane considered above has one kind of the $\mathrm{R}$ - $\mathrm{R}$ charge and no NS-NS charge, and we use the form of $p=3$ in the following.

\section{Solutions of D-brane action}

Here we discuss on the classical solutions of $S_{p+1}$ in the background D-brane(s) which is set parallel to the test brane considering now. The classical equations of $S_{p+1}$ are solved for $p=3$ by adopting the static gauge for the diffeomorphism invariance, for which the world-volume coordinates $\xi^{\mu}$ are equated with the $p+1$ spacetime coordinates as

$$
X^{M}=\xi^{\mu}, \quad M=0,1, \ldots, p .
$$

The remaining coordinates, $X^{m}$, are treated as scalar fields in the brane worldvolume, but we retain only one coordinate among them as a field which is responsible for the configurations of the brane and it is denoted here by $X(\xi)$. This coordinate is taken in the direction perpendicular to the other brane.

Then we can write $S_{p+1}$ with $p=3$ as follows,

$$
S_{4}=-T_{3} \int d^{4} \xi\left(\frac{1}{H}(\sqrt{D}-1)\right)
$$

where the field-independent constant is subtracted from the above lagrangian density for the brevity, and

$$
\begin{aligned}
D= & \left(1-H \vec{E}^{2}\right)\left(1+H(\nabla X)^{2}\right)+H^{2}(\vec{E} \cdot \nabla X)^{2}-H \dot{X}^{2}+2 H^{2} \vec{E} \cdot(\vec{B} \times \nabla X) \dot{X} \\
& +H \vec{B}^{2}\left(1-H \dot{X}^{2}\right)+H^{2}(\vec{B} \cdot \nabla X)^{2}-H^{2}(\vec{B} \cdot \vec{E})^{2},
\end{aligned}
$$

$$
H=1+2 Q \kappa T_{3} G\left(X_{m}-X\right),
$$

where $X_{m}$ denotes the distance between two branes. The U(1) gauge fields are denoted by the conventional, electromagnetic fields $\vec{E}$ and $\vec{B}$.

\subsection{Solutions in the flat background}

Firstly, we solve the equations for $H=1$ : the case of the infinite distance between the two branes. The solutions are restricted to the static one. In this case, $D$ is written as,

$$
D=\left(1-\vec{E}^{2}\right)\left(1+(\nabla X)^{2}\right)+(\vec{E} \cdot \nabla X)^{2}+\vec{B}^{2}+(\vec{B} \cdot \nabla X)^{2}-(\vec{B} \cdot \vec{E})^{2},
$$

and the following equations are obtained,

$$
\begin{array}{r}
\nabla \cdot\left\{\left[\left(1-\vec{E}^{2}\right) \nabla X+(\vec{E} \cdot \nabla X) \vec{E}+(\vec{B} \cdot \nabla X) \vec{B}\right] / \sqrt{D}\right\}=0, \\
\nabla \cdot\left\{\left[\left(1+(\nabla X)^{2}\right) \vec{E}-(\vec{E} \cdot \nabla X) \nabla X+(\vec{B} \cdot \vec{E}) \vec{B}\right] / \sqrt{D}\right\}=0, \\
\nabla \times\{[\vec{B}+(\vec{B} \cdot \nabla X) \nabla X-(\vec{B} \cdot \vec{E}) \vec{E})] / \sqrt{D}\}=0 .
\end{array}
$$


Since we are interested in isotropic solutions, we take the following ansatz:

$$
\vec{E}=f(r) \hat{r}, \quad \vec{B}=g(r) \hat{r}, \quad \nabla X=X^{\prime}(r) \hat{r},
$$

where $\hat{r}$ represents the unit vector in the radial direction, and a prime denotes a differentiation with respect to $r$. Then, Eqs. (17) and (18) are expressed as

$$
\begin{gathered}
\nabla \cdot\left\{X^{\prime} \sqrt{\frac{1+g^{2}}{1-f^{2}+X^{\prime 2}}} \hat{r}\right\}=0, \\
\nabla \cdot\left\{f \sqrt{\frac{1+g^{2}}{1-f^{2}+X^{\prime 2}}} \hat{r}\right\}=0 .
\end{gathered}
$$

From Eqs. (21) and (22), the function $f$ is proportional to $X^{\prime}$

$$
f=\alpha X^{\prime},
$$

where $\alpha$ is a constant. Since the ansatz (20) satisfies automatically Eq. (19) for any $g$, we cannot determine the function $g$ since the number of the independent equations is reduced to two. So we need further ansatz or assumption to obtain the solution. We now assume that the magnetic field $\vec{B}$ is given by some appropriate form as an arbitrary external field, then we can solve the equation Eq. (21) or (22) as follows,

$$
X(r)=\int_{r}^{\infty} d r \frac{A}{\sqrt{\left(1+g^{2}\right) r^{4}-r_{0}^{4}}}
$$

where $A$ denotes an integral constant and $r_{0}^{4}=\left(1-\alpha^{2}\right) A^{2}$. Here we consider the purely electric $(g=0)$ case, namely without the external magnetic field, then the solution (24) reduces to the BPS state for $\alpha=1$ and to the non-BPS solution given in Ref.[1] for $\alpha \neq 1$.

Next, consider the case adding one more ansatz to Eq. (20) as follows:

$$
g=\beta X^{\prime},
$$

where $\beta(\neq 0)$ is a constant. In this case, the string is considered as the source of both the electric and the magnetic charges, so we call this type as the dyonic string. The equation is solved as

$$
X(r)=\int_{r}^{\infty} d r\left[\frac{-\left\{1-\left(1-\alpha^{2}\right) \frac{A^{2}}{r^{4}}\right\}+\sqrt{\left(1-\left(1-\alpha^{2}\right) \frac{A^{2}}{r^{4}}\right)^{2}+4 \beta^{2} \frac{A^{2}}{r^{4}}}}{2 \beta^{2}}\right]^{1 / 2} .
$$

For $\alpha^{2}+\beta^{2}=1$, this solution reduces to the BPS dyonic solution,

$$
X(r)=\frac{A}{r} .
$$

In this case, the Bogomol'nyi equations are satisfied by the saturation of the bound

$$
E \geq \alpha(\vec{E} \cdot \vec{\nabla} X)+\beta(\vec{B} \cdot \vec{\nabla} X) .
$$

The solutions obtained here are classified in the parameter space $\alpha$ and $\beta$ as shown in Fig. 1.

There are many number of non-BPS dyonic string solutions, and some typical one are shown in the Fig.2 along the line in the parameter space shown in Fig.1. 




Figure 1: The static solutions are classified on $\alpha-\beta$ plane. They are represented by three types of solutions: (A)The pure electric solution, (B)the dyonic solution, and (C)the BPS solution. The symbols of plus denote the points on the line $\beta=1.5 \alpha$ for (a) $\alpha=0.3$, (b) 0.5 , (c) 0.7 and (d) 0.9 .

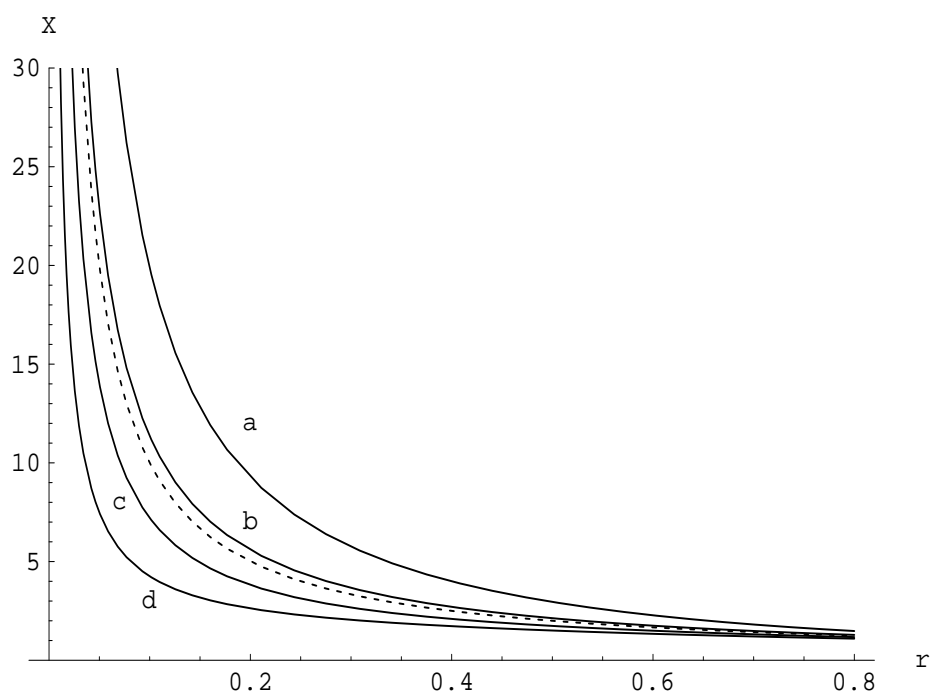

Figure 2: The typical dyonic non-BPS solutions, for $\beta=1.5 \alpha, A=1$. And the curves represent for (a) $\alpha=0.3$, (b) 0.5 , (c) 0.7 and (d) 0.9 in Fig. 1. The boundary conditions are taken as $X(4)=A / 4$ for all curves. 


\subsection{Solutions in the background D-brane(s)}

Here we consider the solutions for the case of $H \neq 1$. In this case, the Wess-Zumino term in the action $S_{4}$ given in Eq. (13) cannot be neglected as in the previous section. To see its important meaning, we notice that $S_{4}$ vanishes for $\vec{E}=\vec{B}=0$. This is a result of the cancellation between the BI and the WZ terms, and it reflects that there is no force between two parallel branes [13]. We therefore expect that the WZ term would play an important role in solving the equations for $H \neq 1$.

Here we solve the equations of motion of $S_{4}$ in the background configuration of the parallel D3-brane(s), so the force is absent for the trivial solution of $\vec{E}=\vec{B}=0$. But it is expected that the non-trivial solutions, which are obtained in the background of flat space-time by solving the BI-action, would be affected by the background configuration. The surviving solutions should be deformed and restricted.

The equations are written as follows:

$$
\begin{gathered}
\frac{\partial H}{\partial X} F_{1}+\nabla \cdot \vec{F}_{2}=0 \\
\nabla \cdot \frac{1}{\sqrt{D}}\left(\left(1+H(\nabla X)^{2}\right) \vec{E}+H[(\vec{B} \cdot \vec{E}) \vec{B}-(\vec{E} \cdot \nabla X) \nabla X]\right)=0 \\
\nabla \times \frac{1}{\sqrt{D}}((\vec{B}+H[(\vec{B} \cdot \nabla X) \nabla X-(\vec{B} \cdot \vec{E}) \vec{E}])=0
\end{gathered}
$$

where $\frac{\partial H}{\partial X}=-4 \tilde{Q} /\left(X_{m}-X\right)^{5}$ and

$$
\begin{aligned}
& F_{1}=\frac{1}{H^{2}}(\sqrt{D}-1)+\frac{1}{2 H \sqrt{D}}\left(\vec{E}^{2}\left(1+H(\nabla X)^{2}\right)-(\nabla X)^{2}\left(1-H \vec{E}^{2}\right)\right. \\
& \left.-2 H\left[(\vec{B} \cdot \nabla X)^{2}+(\vec{E} \cdot \nabla X)^{2}-(\vec{B} \cdot \vec{E})^{2}\right]-\vec{B}^{2}\right) \\
& F_{2}=\frac{1}{\sqrt{D}}\left(\left(1-H \vec{E}^{2}\right) \nabla X+H[(\vec{E} \cdot \nabla X) \vec{E}+(\vec{B} \cdot \nabla X) \vec{B}]\right) .
\end{aligned}
$$

Here the time-dependence of the fields are neglected since the solutions are restricted to the static one. Obviously the effect of the background configuration appears through $H$, and Eqs. (29) $\sim(31)$ are respectively reduced to Eqs. (17) $\sim(19)$ for $H=1$. The most characteristic feature is seen in the first term of the l.h.s. of Eq. (29). And the WZ term is also included in $F_{1}$ of this term. For $\tilde{Q}=0$, this term disappears, and the second term reduces to Eq. (17) given in the flat background. The first term therefore represents a newly appeared constraint due to the non-trivial background configuration. Due to this term, the above equations can be solved with ansatzs (20) and (23) only since the new constraint determines the magnetic field $g(r)$ as seen below.

We solve the above equations by taking the ansatz (20) used in the previous subsection. Then the above equations are rewritten as follows:

$$
\frac{\partial H}{\partial X} F_{1}+\nabla \cdot\left(\frac{X^{\prime}}{\sqrt{D}}\left(1+H g^{2}\right) \hat{r}\right)=0
$$




$$
\begin{gathered}
\nabla \cdot\left(\frac{f}{\sqrt{D}}\left(1+H g^{2}\right) \hat{r}\right)=0, \\
\nabla \times\left(\frac{f}{\sqrt{D}}\left(1+H\left(X^{\prime 2}-f^{2}\right)\right) \hat{r}\right)=0,
\end{gathered}
$$

where

$$
\begin{gathered}
F_{1}=\frac{1}{2 H^{2} \sqrt{D}}\left(2(D-\sqrt{D})+H\left(f^{2}-X^{\prime 2}-g^{2}\right)+2 H^{2} g^{2}\left(f^{2}-X^{\prime 2}\right)\right) \\
D=\left(1+H g^{2}\right)\left(1+H\left(X^{\prime 2}-f^{2}\right)\right) .
\end{gathered}
$$

With the same way as in the previous subsection, the number of equations is reduced to two since Eq. (36) is satisfied by any functional form of $f, g$ and $X$.

Then we need one more ansatz which gives one relation among these three functions. To find similar solutions to the one obtained in the previous subsection, we take the following ansatz

$$
f=\alpha X^{\prime}
$$

It should be noticed that this was not an ansatz but a solution of the equations in the previous subsection. Under this ansatz, Eq. (35) is solved as

$$
\frac{f}{\sqrt{D}}\left(1+H g^{2}\right)=\frac{A_{2}}{r^{2}},
$$

where $A_{2}$ is an integral constant. The Eq. (34) is rewritten as

$$
F_{1}=0
$$

since the second term of this equation vanishes, i.e. $\nabla F_{2}=0$. We notice that the above equation (41) does not appear in the case of the flat background. As a result, the magnetic field $g(r)$ can not be determined. In the present case, we can obtain the following solution due to Eq. (41),

$$
X=\frac{A_{2}}{r}, \quad f=-\alpha \frac{A_{2}}{r^{2}}, \quad g=-\sqrt{1-\alpha^{2}} \frac{A_{2}}{r^{2}} .
$$

This solution represents the dyonic string with both electric and magnetic charges, and the BPS bound is saturated by this solution since it is represented as

$$
\vec{E}=\cos (\theta) \nabla X, \quad \vec{B}=\sin (\theta) \nabla X
$$

by the parametrization $\cos \theta=\alpha(\leq 1)$. Here, we notice the following points: (i) The ansatz (39), which yields a non-BPS solution in the case of the flat background, leads to the BPS solution due to the Eq. (41). (ii) The form of the BPS solution obtained is independent on the parameter of the background since it is not deformed by the background configurations. (iii) The effect of the background configuration appears indirectly through the induced world-volume metric in the brane action. Namely, $r$ has a minimum value, $r_{0}=A_{2} / X_{m}$ [9], where the solution touches the opposite brane(s) since $X\left(r_{0}\right)=X_{m}$. At this point, the proper distance in the world-volume of the brane becomes infinite since the induced metric diverges. Then 
we arrive at the configuration of the dyonic string which connects two branes with a finite distance.

From the fact (iii), the energy of the string part is obtained as a finite value by integrating the energy density of the system in the range $r_{0}<r<\infty$ as follows,

$$
E=4 \pi A_{2} T_{3} X_{m}
$$

which represent the energy of the string of the length $X_{m}$ with the tension of $4 \pi A_{2} T_{3}$. It is expected that this object with a finite energy would appear as a dyon in the Yang-Mills theory.

\section{Non-BPS solutions}

We now turn to the non-BPS solutions. As seen in the subsection 4.1, the nonBPS solutions obtained in the flat space are separated into two groups from their property. One is the pure electric string which connects to the anti-brane to form a bound state. The second is the dyonic string which could arrive at the other brane(s) at the infinite distance. In the case of the finite distance also, these two types of solutions can be found by taking other ansatz than the one given in the previous section, (39).

\subsection{Pure electronic case}

First we consider the case of the pure electric solution. This is obtained by taking the following ansatz,

$$
g=0
$$

In this case, we can obtain the following equation for $X$ from Eqs. (34) and (40),

$$
X^{\prime \prime}+\frac{2}{r} X^{\prime}-\frac{X^{\prime}}{2}(\ln D)^{\prime}=\frac{2 \tilde{Q}(\sqrt{D}-1)^{2}}{X^{5} H^{2}}
$$

where

$$
D=\frac{1+H X^{\prime 2}}{1+H\left(A_{2} / r^{2}\right)^{2}} .
$$

It is easily seen that Eq. (46) is reduced to the equation of the flat background in the limit of $\tilde{Q}=0(H=1)$. In fact, we obtain the following solution for $\tilde{Q}=0$,

$$
X^{\prime}=\frac{c_{1} / r^{2}}{\sqrt{1-c_{2} / r^{4}}},
$$

where $c_{1}$ and $c_{2}$ are constants depending on the boundary conditions. This is equivalent to the solution (24).

While Eq. (46) for $H \neq 1$ is highly nonlinear, so it is difficult to solve it analytically. Then we give the solutions numerically. 


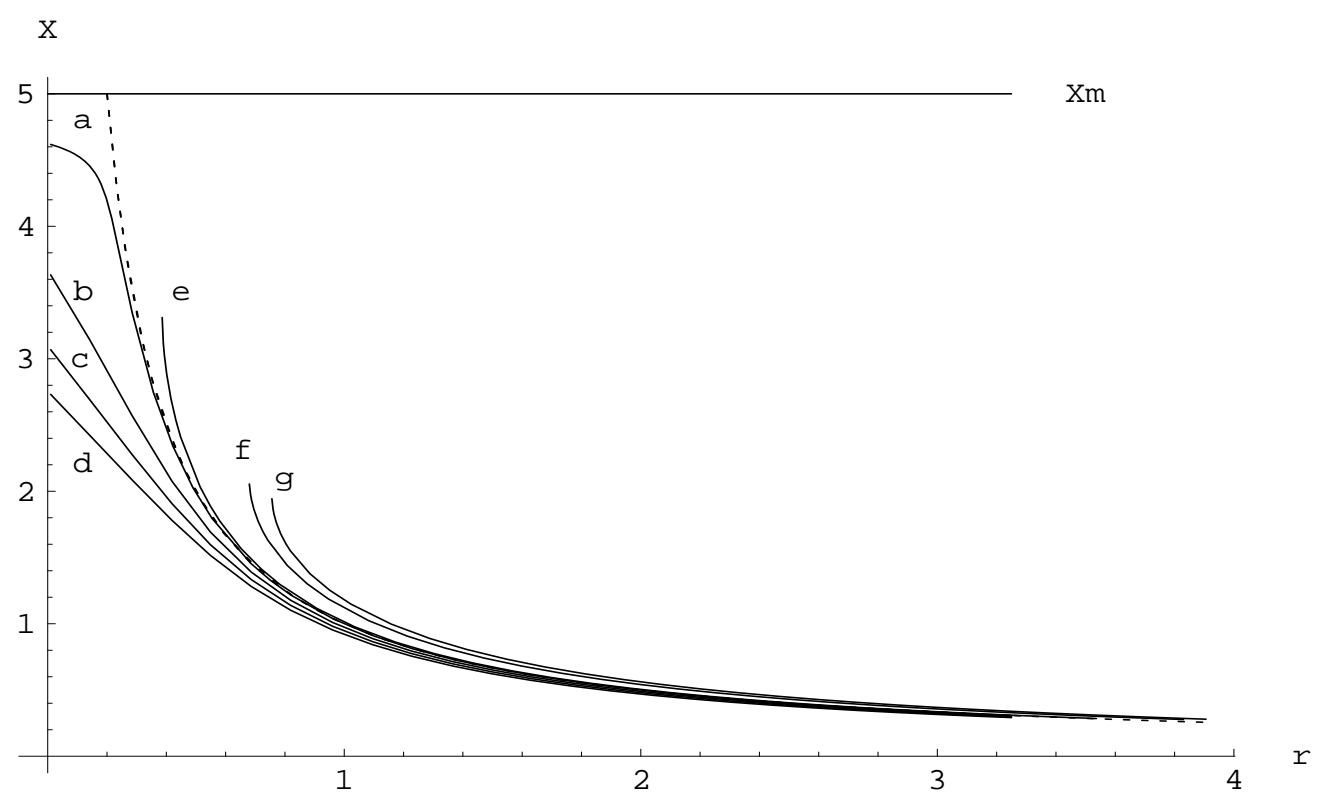

Figure 3: The typical two types of non-BPS solutions, for $\tilde{Q}=1, X_{m}=5, A_{2}=1$. The boundary conditions are taken as $X(10)=0.1$ and (a) $X^{\prime}(10)=-0.00974$, (b) -0.00948 , (c) -0.00923 , (d) -0.00898 for the first type, and (e) $X^{\prime}(10)=-0.0101$, (f) -0.011 , (g) -0.0115 for the second type.

The resultant solutions are shown in Fig. 3, where the BPS solution, $X=A_{2} / r$, is also shown by the dotted line for the comparison. In the Fig.3, two types of nonBPS solutions are seen. They are obtained here by giving the boundary conditions at an appropriate point $\left(r=r_{B}\right)$ as

$$
X\left(r_{B}\right)=\frac{A_{2}}{r_{B}}, \quad X^{\prime}\left(r_{B}\right)=-\gamma \frac{A_{2}}{r_{B}^{2}},
$$

where $\gamma$ is a parameter. The solutions of the first group $(a) \sim(d)$ are obtained for $\gamma<1$, and the second group $(e) \sim(g)$ are for $\gamma>1$. We notice that the BPS solution is obtained for $\gamma=1$. In this sense, the BPS solution is the critical one which separates the two types of solutions.

The first type of solution covers all region of $r$, and it ends at $r=0$ with finite value of $X(0)$ and negative value of $X^{\prime}(0)$. So the shape of this configuration has a cusp at $r=0$ and the configuration is singular at this point.

The second type of solution is bounded as $r \geq r_{1}$, where $r_{1}$ varies depending on the boundary conditions and $X^{\prime}\left(r_{1}\right)=\infty$. Due to this property, the solution can be connected to the other half with the opposite orientation [1] to make the bound state of brane and anti-brane.

These solutions have the same qualitative properties with the one obtained in the flat background. However they are affected by the non-trivial background in the present case. The most prominent influence is seen in the fact that these solutions could not reach at the position of the other brane(s), $X=X_{m}$. This is understood as follows. From Eqs. (38) and (40), we obtain

$$
f^{2}=\frac{H^{-1}+X^{\prime 2}}{H^{-1}\left(r^{2} / A_{2}\right)^{2}+1} .
$$


Then we find the relation $f=X^{\prime}$ at $X=X_{m}$, where $H^{-1}=0$, and we obtain the BPS solution from this condition. This implies that only the BPS solution can arrive at $X=X_{m}$. So there is no non-BPS electric string state, which connect two parallel branes with a finite energy. Such a object is restricted to the BPS saturated strings.

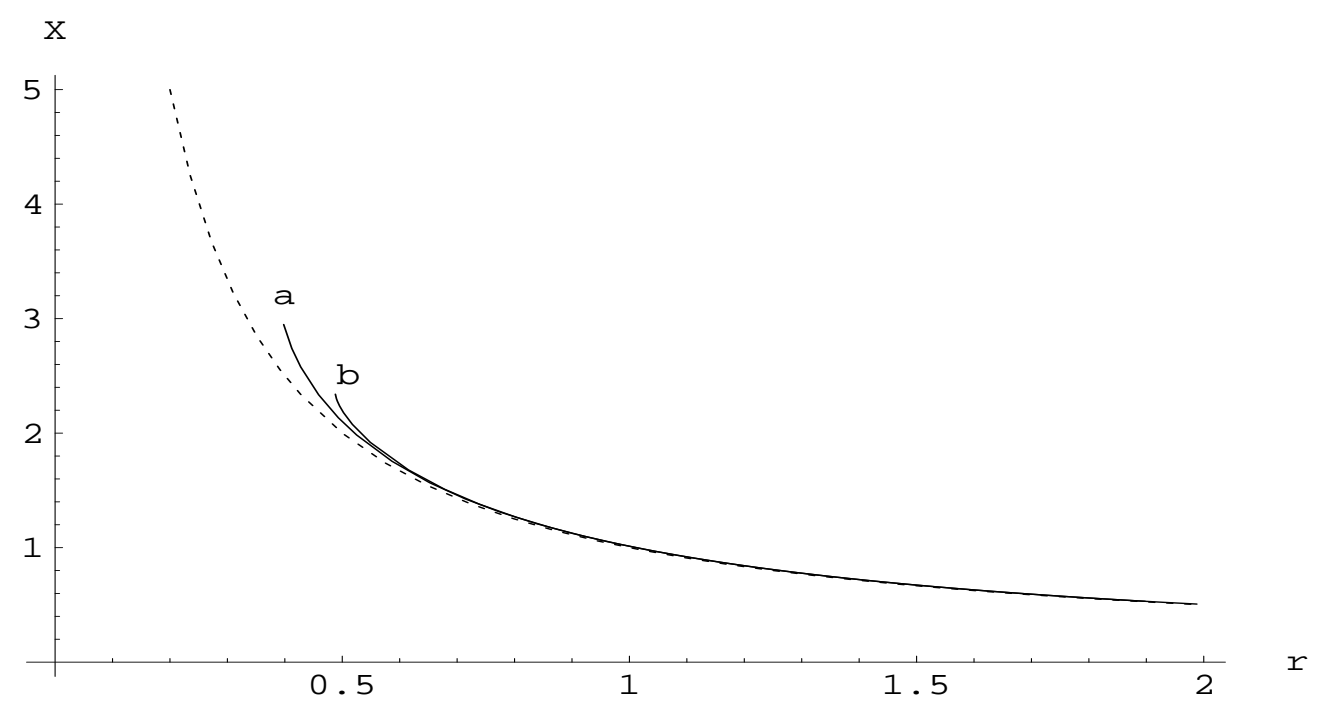

Figure 4: The typical non-BPS solutions for $X_{m}=5, A_{2}=1$, (a) $\tilde{Q}=1$ and (b) $\tilde{Q}=70$ with the boundary condition $X(10)=0.1$ and $X^{\prime}(10)=-0.0101$.

As for the $\tilde{Q}$ dependence of the solutions, we can see the typical effect from the solutions as shown in Fig. 4, where the second type of solutions for two different $\tilde{Q}$ value are given. The $\tilde{Q}$ dependence is small, but it can be seen near the end point of the solution. The larger $\tilde{Q}$ becomes the wider the radius of the tube grows, and the end point of the string configuration goes back. This implies that the bound state of the brane and anti-brane would be pushed to vanish through the annihilation of them near the branes.

\subsection{Dyonic case}

Next, we consider the dyonic non-BPS string configurations under the D-brane background. The equation for this solution is obtained by taking the ansatz (25), $g=\beta X^{\prime}$, which is given in the section 3.1 to obtain the dyonic solution in the flat background. In the present case, Eq. (23) is not used differently from the the case of section 3.1. In terms of this ansatz (25) and Eq. (40), which is the solution of (35), we obtain

$$
f^{2}=\frac{1+H X^{\prime 2}}{1+H\left[\beta^{2} X^{\prime 2}+\left(A_{2} / r^{2}\right)^{2}\right]}\left(\frac{A_{2}}{r^{2}}\right)^{2} .
$$

Substituting Eq. (51) and (25) into Eq. (34), we can solve Eq. (34) with respect to $X(r)$ by rewriting it as the differential equation of $X(r)$. We solve the differential equation numerically.

We firstly discuss the dyonic solutions which are corresponding to the solutions $(g)$ in Fig.3 at the limit of $\beta=0$. Namely we take the same boundary conditions 
with the one of $(g)$ in Fig.3 in solving the equation considered here for $\beta \neq 0$. In Fig.5, the solutions are shown for various values of $\beta$. The $\beta$-dependence of the solutions is seen from the Fig.5, and the behaviors are similar to the case of the flat background.

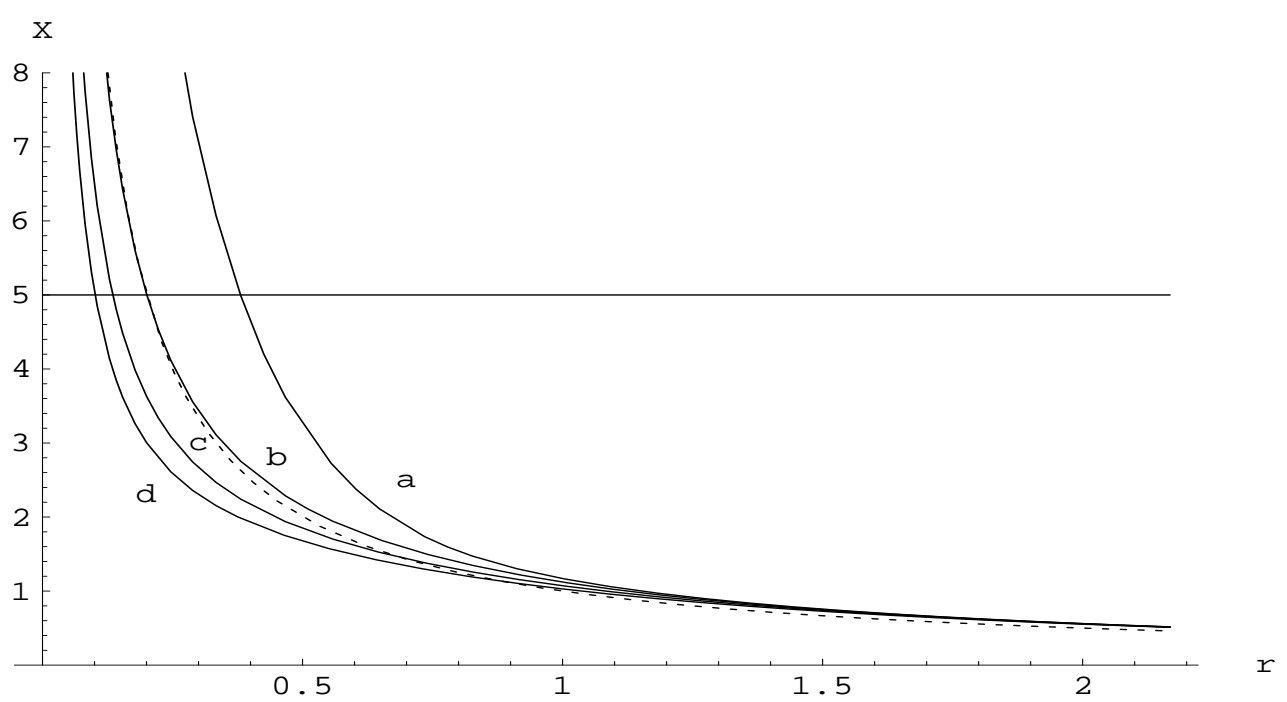

Figure 5: The typical dyonic non-BPS solutions for $X_{m}=5, A_{2}=1$, (a) $\beta=0.2$, (b) $\beta=0.6$, (a) $\beta=1.0$ and (b) $\beta=1.4$ with the boundary condition $X(10)=0.1$ and $X^{\prime}(10)=-0.0115$.

These solutions exceed $X_{m}$ which was the bound for the non-BPS solution of $\beta=0$. This property is also seen in the case of the flat background, where this configuration extends to $X=\infty$ at $r=0$. But the shapes of this configurations are affected by the D-brane background. This point is different from the case of the flat background. This is seen from the results shown in Fig.6, in which $\tilde{Q}$-dependence for the solution is presented. Although the $\tilde{Q}$-dependence is small, we can see it
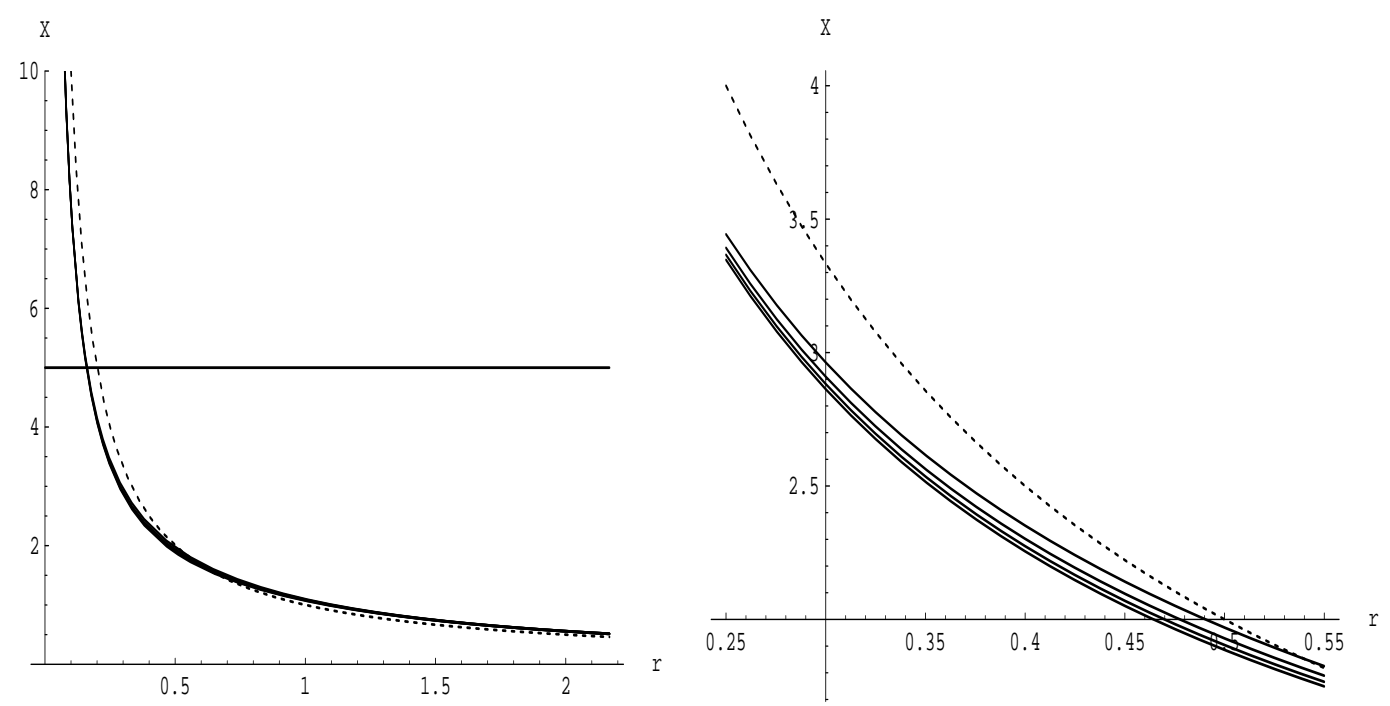

Figure 6: The $\tilde{Q}$-dependence of the dyonic non-BPS solutions for $X_{m}=5, A_{2}=1, \beta=0.8$ with the boundary condition $X(10)=0.1$ and $X^{\prime}(10)=-0.0115$. From the above curve, the curves for $\tilde{Q}=1,101,201,301$ are shown. 
near the neck $(0.2<r<6)$ of the throat by extending the scale of $X(r)$. This $\tilde{Q}$-dependence is the main difference from the BPS solutions which are independent on the background.

We should notice the following fact. For any solution, the metric of the worldvolume action is determined from the same D-brane background, and it has a singularity at $X=X_{m}$. So the configuration should be cut at this point, then we can say that the configuration obtained here represents the dyonic string which connects two D-branes with a finite distance. But these solutions are not the BPS states, so some dynamical corrections would modify the configurations obtained here. This is a dynamical problem, which would be related to the non-supersymmetric Yang Mills theory. This point is open here.

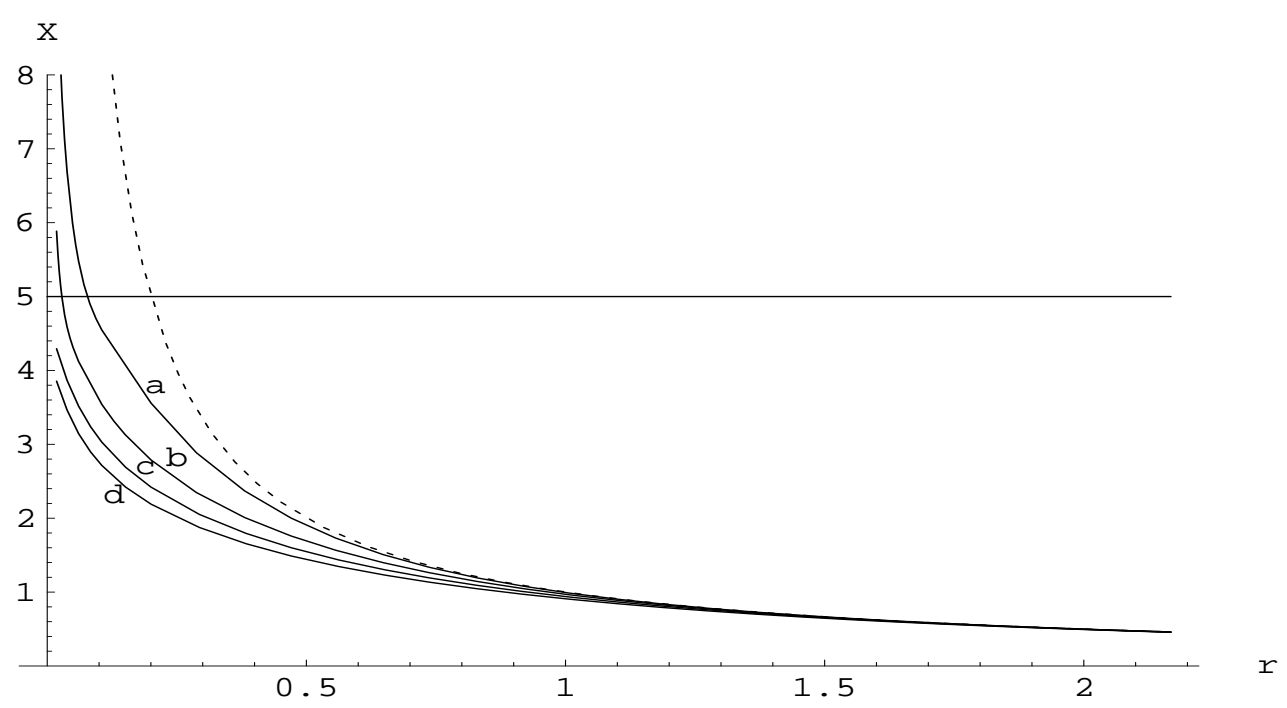

Figure 7: The $\beta$-dependece of the dyonic non-BPS solutions for $X_{m}=5, A_{2}=1, \tilde{Q}=1$ with the boundary condition $X(10)=0.1$ and $X^{\prime}(10)=-0.009974$. The curves for (a) $\beta=0.2$, (b) 0.6 , (c) 1.0 and $(\mathrm{d}) 1.4$ are shown.

Finally, we comment on the solutions of $\beta \neq 0$ which are reduced to the solutions belonging to the group of $(a) \sim(d)$ of Fig. 3 for $\beta=0$. The results are shown in the Fig. 7 for various values of $\beta$. In this case, the qualitative behavior of the solutions is the same with the one of $\beta=0$. But we should notice that there is no bound in principle at $X=X_{m}$.

\section{Conclusions}

We have given the solutions of the world-volume action of a D3-brane which is placed parallel to the background D3-brane(s). The equations are firstly solved in the flat background, and two kinds of non-BPS solutions are shown, i.e. the pure electric and the dyonic solutions. They smoothly approaches to the BPS solutions in a special limit of the parameters.

Both the BPS and non-BPS solutions are also obtained in the D-brane(s) background. The BPS solutions has the same functional form with the one obtained in the flat background and it can extend to infinity. But it should be bounded at the 
position of the opposite brane(s) since the proper distance in the world-volume of the brane becomes infinite there. As a result, a finite energy of this BPS state is obtained.

On the other hand, both types of non-BPS solutions are affected by the D-brane background. Especially the electric-type solutions can not arrive at the opposite brane(s) since they are pushed back by the background configurations. In this sense, this type of non-BPS solutions can not be considered as strings which connect two branes. One of these non-BPS solutions can be interpreted as the half-part of the bound state of the brane and anti-brane as in the case of the flat background. It is observed that the distance between the brane and the anti-brane of this bound state becomes shorter when it nears the background branes.

Although the configurations of the dyonic non-BPS solutions are also affected by the D-brane background, they could extend over $X=X_{m}$ and $X$ becomes infinite at $r=0$. On the other hand, the induced-metric of the world-volume action of the D-brane is independent on the solutions, so the configurations of the solutions are bounded at $X=X_{m}$ as in the case of the BPS solutions. Then we arrive at the conclusion that the configuration of non-BPS dyonic string connects two D-branes with a finite distance and with a finite energy. This configuration however receives a dynamical correction because of no supersymmetry, and this would be related to the dynamics of the non-supersymmetric Yang -Mills dynamics.

\section{References}

[1] C.G. Callan and J.M. Maldacena, "Brane Dynamics from the Born-Infeld Action," hep-th/9708147

[2] G.W. Gibbons, "Born-Infeld particles and Dirichlet p-branes," Nucl. Phys. B514, 603 (1998), hep-th/9709027.

[3] A. Hashimoto, "The shape of branes pulled by strings," Phys. Rev. D57, 6441(1998).

[4] D. Bak, J. Lee and H. Min, "Dynamics of BPS States in the Dirac-Born-Infeld Theory," hep-th/9806149.

[5] K.G. Savvidy, "Brane Death via Born-Infeld String," hep-th/9810163.

[6] Y.Imamura, "Supersymmetries and BPS Configurations on Anti-de Sitter Space," hep-th/9807179.

[7] C.G. Callan, A. Guijosa and K.G. Savvidy, "Baryons and String Creation from the Fivebrane Worldvolume Action," hep-th/9810093.

[8] C.G. Callan, A. Guijosa, K.G. Savvidy and O. Tafjord, "Baryons and Flux Tubes in Confining Gauge Thories from Brane Actions," hep-th/9902197. 
[9] J.P. Gauntlett, C. Koehl, D, Mateos, P.K. Townsend and M. Zamaklar, "Finite energy Dirac-Born-Infeld monopoles and string junctions," hep-th/9903156

[10] J.M. Camino, A.V. Ramallo and J.M. Sanchez de Santos, "Worldvolume Dynamics of D-branes in a D-brane Background," hep-th/9905118.

[11] M. J. Duff, R. R. Khuri and J. X. Lu, Phys. Rep., 259, 213(1995).

[12] G. T. Horowitz and Strominger, Nucl. Phys., A360, 197(1991).

[13] J.Polchinski., Phys. Rev. Lett., 75, 141(1997), hep-th/9711106 